\title{
Towards fully-resolved PIV measurements in high Reynolds number turbulent boundary layers with DSLR cameras
}

\author{
C.M. de Silva · K. Grayson · S. Scharnowski · C.J. Kähler • \\ N. Hutchins - I. Marusic
}

Received: date / Accepted: date

\begin{abstract}
In this study, we describe a multi-camera large field-of-view (FOV) planar-PIV experiment to capture the wide range of scales that coexist in high Reynolds number turbulent boundary layers. The proposed measurements are designed to capture spatial flow features over a greater range than current common practices, and at significantly lower cost. With this goal in mind, specialist PIV cameras are substituted with modern consumer full-frame digital cameras, which are typically available at a fraction of the cost, with higher resolution sensors. These cameras are configured to capture single-frame double-exposed images (DE-PIV), but at a much higher spatial resolution than what is available from specialist PIV cameras that capture double-frame single-exposure images (SE-PIV). This work discusses a set of simulations and experiments to quantitatively assess the quality of the PIV velocity fields from these two approaches for large field-of-view measurements. Our findings confirm that despite the known loss-of-accuracy associated with DE-PIV, the use of high-resolution cost-effective consumer cameras provides an economically feasible PIV solution with the necessary performance and accuracy for high spatial range measurements in wall-bounded turbulent flows.
\end{abstract}

Keywords Particle Image Velocimetry $\cdot$ Double Exposure $\cdot$ Wall Turbulence $\cdot$ DSLR Camera

\section{Introduction}

Over the last two decades, particle image velocimetry (PIV) has become increasingly popular for carrying out accurate, multi-component, multi-dimensional spatial measurements in turbulent flows [Adrian and Westerweel, 2011, Raffel et al., 2017]. Although one can obtain higher signal-to-noise ratios and longer time-averaged signals from single point measurement techniques (e.g. hot-wire anemometry [Bruun, 1995]), PIV provides spatial derivatives, flow visualisations and spatial correlations without disturbing the flow or fluid properties. To date, PIV remains one of the few techniques that offer such spatial information in rapidly evolving flows, and at a level of detail that was once considered achievable only in numerical simulations. Among the many applications of PIV, the study of turbulent flows still stands as one of the most demanding due to the wide range of length and time scales that coexist. A characteristic measure of

C.M. de Silva $\cdot$ K. Grayson · N. Hutchins · I. Marusic

Department of Mechanical Engineering,

University of Melbourne,

VIC 3010, Australia.

E-mail: desilvac@unimelb.edu.au

S. Scharnowski · C. J. Kähler

Institute of Fluid Mechanics and Aerodynamics,

Bundeswehr University Munich,

Neubiberg, Germany. 
this length-scale range in turbulent boundary layers is the friction Reynolds number, or Kármán number, $R e_{\tau}=\delta U_{\tau} / v$, where $U_{\tau}$ is the friction velocity, $\delta$ is the boundary layer thickness and $v$ is the kinematic viscosity. $R e_{\tau}$ is proportional to the ratio of the largest and smallest length scales in the flow. Consequently, the scale separation between the largest and smallest length scales increases with $R e_{\tau}$. Presently, PIV experiments seeking to resolve a wide range of scales require large infrastructure expenditure for specialist equipment and typically demand the use of large multi-camera arrays (for recent works in boundary layers see de Silva et al. [2014], Knopp et al. [2014], Reuther et al. [2015], Buchmann et al. [2016]). Therefore, high Reynolds number PIV is often limited by the available dynamic spatial range (DSR), a measure of the ratio between the maximum and minimum resolvable length-scales [Adrian, 1997].

One early attempt that demonstrated the significance of high DSR PIV measurements in turbulent boundary layer research is the seminal work of Adrian et al. [2000]. To achieve a high DSR, they utilised a rather laborious process of digitising and post-processing double-exposed large-format photographic film. This approach limited the number of frames that could be acquired, but provided a high DSR to resolve the range of scales that coexist at the moderate Reynolds number studied. A decade after the work of Adrian et al. [2000], PIV has been applied extensively in turbulent flows. However, as summarised in a review article by Westerweel et al. [2013] (see also Raffel et al. [2017]), although modern PIV techniques have led to considerable advancements in experimental capability (higher acquisition rates and volumetric information), these modern techniques are yet to match the DSR from analogue photographic film PIV acquired over two decades ago.

This paper outlines the development and testing of a cost-effective imaging system by substituting specialist PIV cameras with modern consumer cameras, which typically are a fraction of the cost $(\approx 1 / 10$ th) of a specialist PIV camera per megapixel. These cameras also generally provide a higher number of pixels per camera but are not capable of achieving the interframing time and shutter speeds necessary for singleexposure PIV (SE-PIV), where two successive exposures are acquired on separate frames. It is possible, however, to use standard consumer cameras for single-frame double-exposure PIV (DE-PIV, where two exposures are captured on a single frame) techniques to obtain well-resolved, large FOV experiments with a high DSR. There are known drawbacks of DE-PIV, such as directional ambiguity (for two-pulse PIV) and an increased noise floor (necessitating larger interrogation windows to maintain accuracy). However, in the present application (wall-bounded flows), flow reversals are a rare event and are confined to locations very close to the wall, in the viscous layer [Kähler et al., 2012a]. Therefore, the issue of directional ambiguity is not critical in this study. It is worth noting that past works impacted by directional ambiguity have attempted to overcome this drawback by conducting colour PIV with light sources of different wavelengths so that two frames can be separated from a single exposure [Funatani et al., 2013, Wang et al., 2017]. Other solutions include using image shifting techniques [Raffel and Kompenhans, 1995] and double pulse laser systems with polarisation sensitive optics [Adrian, 1986] to resolve direction ambiguity in DE-PIV.

The efficacy of DE-PIV techniques and their corresponding noise characteristics have been extensively examined against SE-PIV methods by Keane and Adrian (see Keane and Adrian [1990, 1992]). In the present work, we focus our attention on the feasibility of a cost-effective imaging system using DE-PIV for large-FOV, high DSR measurement in a high Reynolds number turbulent boundary layer. Accordingly, we examine a set of simulations and experiments tailored for large-FOV PIV measurements in wall-bounded turbulence. Through this analysis, we also quantify the accuracy of DE-PIV and SE-PIV using recent PIV processing algorithms [Adrian and Westerweel, 2011, Raffel et al., 2017], which have been developed after most PIV practitioners had fully adopted SE-PIV.

Throughout this paper, the coordinate system $x, y$ and $z$ refer to the streamwise, spanwise and wallnormal directions, respectively. Corresponding instantaneous streamwise, spanwise and wall-normal velocities are represented by $\widetilde{U}, \widetilde{V}$ and $\widetilde{W}$, respectively, with the corresponding velocity fluctuations given by lower case letters. Overbars indicate averaged quantities, and the superscript + refers to normalisation by inner scales. For example, we use $l^{+}=l U_{\tau} / v$ for length and $\widetilde{U}^{+}=\widetilde{U} / U_{\tau}$ for velocity, where $U_{\tau}$ is the friction velocity and $v$ is the kinematic viscosity of the fluid. 


\begin{tabular}{|c|c|c|c|c|c|}
\hline Case / Symbol & Processing & $\begin{array}{l}\text { Sensor resolution } \\
\text { megapixels (MP) }\end{array}$ & $\begin{array}{l}\text { Interrogati } \\
\text { pixels }\end{array}$ & $\begin{array}{l}\text { window size } \\
\text { wall units }\end{array}$ & 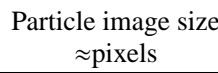 \\
\hline Case $1-\boldsymbol{\nabla}$ & SE-PIV & 11 & $32 \times 32$ & $50 \times 50$ & 3 \\
\hline Case 2 - $\square$ & DE-PIV & 11 & $32 \times 32$ & $50 \times 50$ & 3 \\
\hline Case 3 - $\Delta$ & DE-PIV & 44 & $64 \times 64$ & $50 \times 50$ & 6 \\
\hline
\end{tabular}

Table 1 Parameters for single-exposure (SE) and double-exposure (DE) PIV simulations. All simulations are run on a wall-parallel (xy) plane using a DNS database at $R e_{\tau} \approx 2500$ to displace the synthetic particles.

\section{Simulation study}

In order to examine the feasibility of using DE-PIV in high Reynolds number wall-bounded flows, an assessment of the technique's performance is undertaken with numerical simulations of PIV experiments. The use of numerical simulations enables control over all measurement parameters, while still considering experimentally realistic turbulent flows [Kähler et al., 2016]. Following prior works (see Worth et al. [2010], de Silva et al. [2012a], Grayson et al. [2018]), we use a direct numerical simulation (DNS) database of a turbulent boundary layer at $R e_{\tau} \approx 2500$ [Sillero et al., 2013] to provide realistic velocity fields to displace the synthetic particle images. For the present purpose, ten independent volumetric velocity fields from the DNS database are employed to generate synthetic planar-PIV particle images on a wall-parallel plane in the logarithmic region $(z / \delta \approx 0.1)$. Processing of the synthetic PIV images is performed by an in-house PIV package [de Silva et al., 2012a, Grayson et al., 2018], which uses a cross-correlation algorithm with multi-grid [Willert, 1997] and window deformation applied to each pass [Scarano, 2001]. Key parameters of the simulations are summarised in table 1, which include the sensor resolution (in megapixels) and the final interrogation window size. In the present work, case 1 is used as a baseline SE-PIV reference, with comparable conditions to prior large field-of-view PIV measurements [de Silva et al., 2015]. While these parameters are not necessarily optimal, they are representative of typical PIV measurements tailored to capture large fields-of-view. The DE-PIV simulation parameters for case 2 are matched to the SE-PIV simulations in case 1, while parameters for case 3 are chosen to match an experimental DE-PIV database to be detailed in section 3 .

To visualise the accuracy of the velocity fields from the DE-PIV simulations, figure 1(a) presents colour contours of instantaneous streamwise velocity from a representative SE-PIV and DE-PIV simulation (case 1 and 2) as compared to the original DNS data. Qualitatively, both simulations show good agreement with the reference DNS velocity field (left column) and they appear to be visually indistinguishable from each other. In a similar fashion, to visualise the accuracy of the velocity gradients, figure 1(b) presents colour contours of wall-normal vorticity $\left(\omega_{z}\right)$, where good agreement between the SE- and DE-PIV simulations are exhibited. Collectively, these observations suggest that the use of DE-PIV has, in the present case, not introduced a substantial penalty in accuracy for both the velocity magnitude and its spatial gradients.

In order to quantify the inaccuracy associated with each set of simulations, we compute a probability density function (p.d.f.) of the difference in velocity components between the PIV simulations $(\widetilde{U}, \widetilde{V})$ and the DNS $\left(\widetilde{U}_{D N S}, \widetilde{V}_{D N S}\right)$ velocity fields. We note that for all subsequent comparisons, the DNS velocity fields are spatially filtered to account for the spatial attenuation of the interrogation window size and laser sheet thickness [Lee et al., 2016]. The results are shown in figure 2, which confirms that at matched sensor resolution and interrogation window size (pixels), SE-PIV ( $\mathbf{\nabla}$ symbols) provides superior accuracy (a higher peak about zero) than DE-PIV ( $\square$ symbols). However, applying DE-PIV to images with more pixels ( $\Delta$ symbols) that reflect the sensor resolutions of current digital single-lens reflex (DSLR) cameras, while maintaining the same field-of-view and interrogation window size in viscous units / physical dimensions, improves accuracy toward that obtained by the lower resolution SE-PIV simulations. Although the highresolution DE-PIV cannot totally match SE-PIV in error performance, the double-exposure approach allows the use of consumer cameras that are a fraction of the cost of specialist PIV cameras, without any significant penalties in accuracy.

We note that even though certain consumer cameras are capable of achieving even higher sensor resolution (higher number of pixels), further increases in pixel density do not result in significant gains (at a fixed FOV), since the particle image size is already sufficient to resolve the particle locations (see Adrian and Westerweel [2011], Kähler et al. [2012b]). However, the increased number of pixels (which is readily 

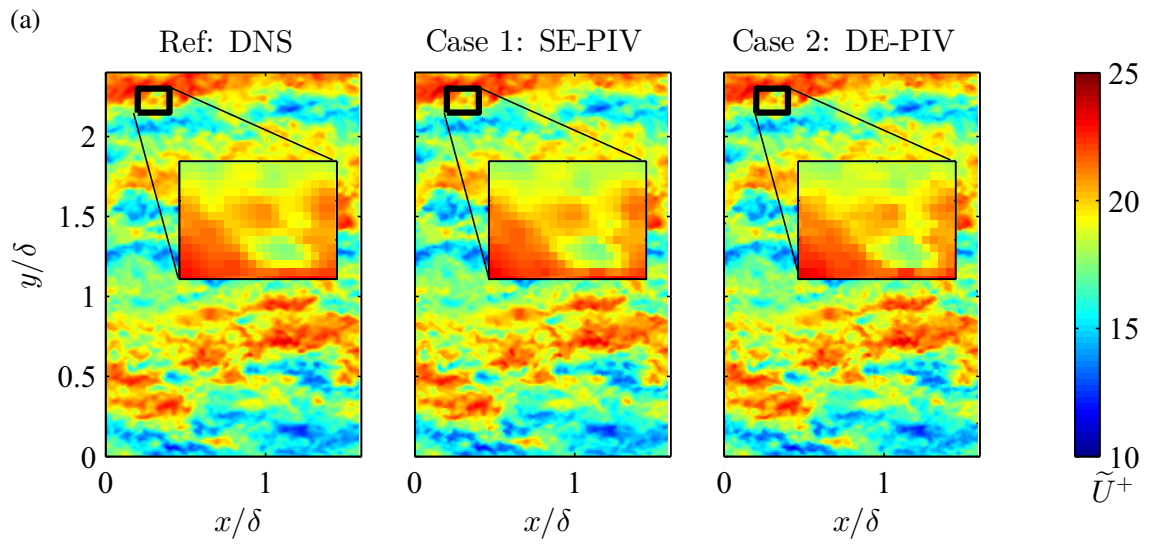

(b)
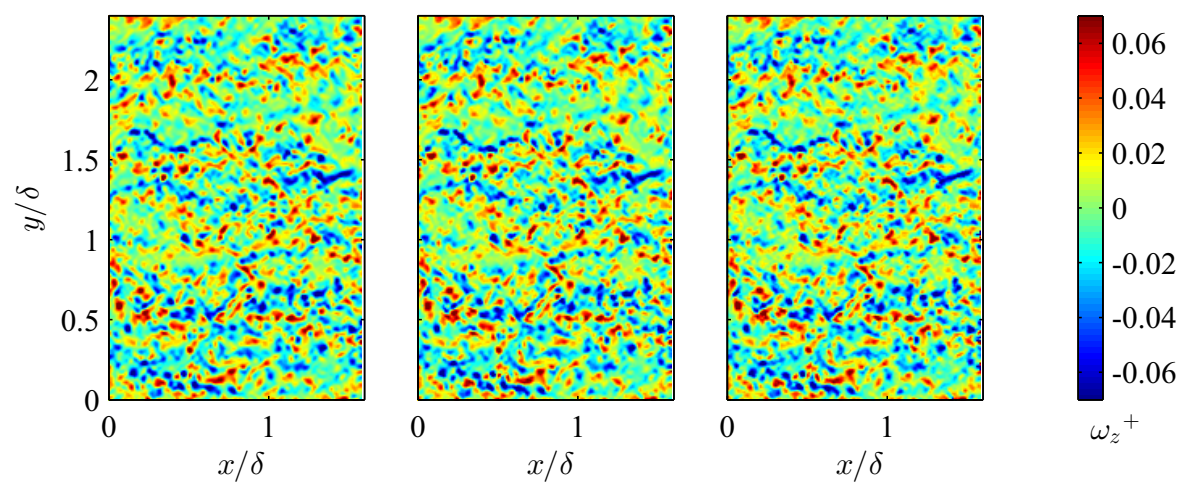

Fig. 1 (a) Colour contours of streamwise velocity from the (left) reference DNS database, (middle) Case 1: SE-PIV and (right) Case 2: DE-PIV simulations. Results are presented on a streamwise/spanwise plane in the logarithmic region of a turbulent boundary layer at $R e_{\tau} \approx 2500$. (b) wall-normal vorticity field corresponding to (a).
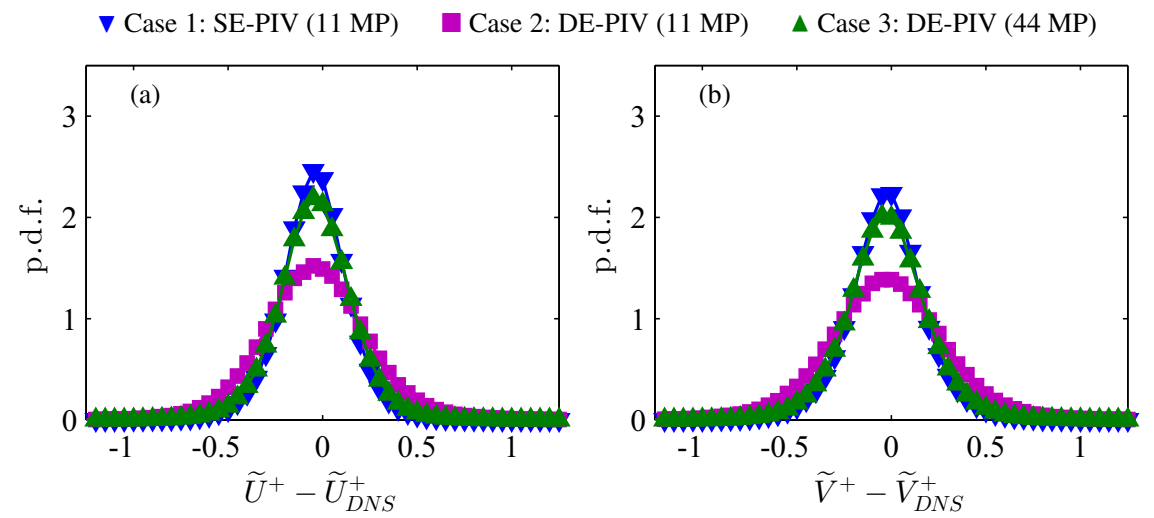

Fig. 2 Probability density function (p.d.f.) of the error of (a) streamwise $\left(\widetilde{U}^{+}-\widetilde{U}_{D N S}^{+}\right)$and (b) spanwise $\left(\widetilde{V}^{+}-\widetilde{V}_{D N S}^{+}\right)$velocity components from the PIV simulations. Results are normalised in viscous units, where $\mathbf{\nabla}, \mathbf{a}$ and $\boldsymbol{\Delta}$ symbols correspond to simulation cases 1 to $3 . \widetilde{U}_{D N S}$ and $\widetilde{V}_{D N S}$ correspond to the velocity fields from the DNS database used to displace the synthetic particles, which are spatially filtered to match the spatial resolution of the PIV simulations.

available from consumer cameras) enables us to capture larger FOVs with the same number of cameras, while still maintaining an equivalent spatial resolution (or interrogation window size in viscous units). Such a scenario is of particular benefit for high Reynolds number experiments, where a large FOV is essential to capture the larger-scale-motions that are $O(\delta)$, while maintaining adequate fidelity to resolve the smaller scales that also coexist.

The absolute error of the velocity components (see figure 2) is a good diagnostic to assess the quality of the PIV simulations, however, this comparison cannot be applied to experimental data. Therefore, to further 

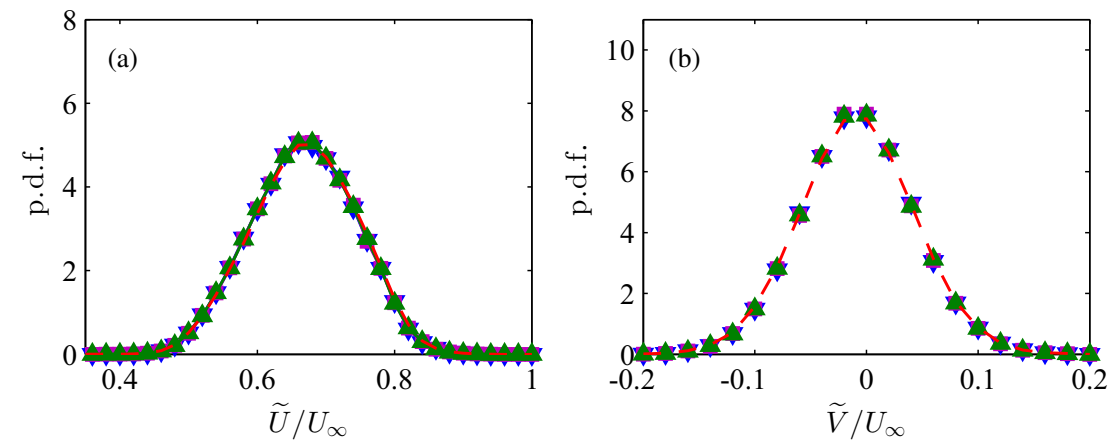

Fig. 3 P.d.f. of the (a) streamwise $(\widetilde{U})$ and (b) spanwise $(\widetilde{V})$ velocity components from the simulations. Results are presented at $R e_{\tau} \approx 2500$ in the logarithmic region of the boundary layer. $\mathbf{\nabla}, \mathbf{\square}$ and $\boldsymbol{\Delta}$ symbols correspond to simulation cases 1 to 3 , which are summarised in table 1 . The reference profile shown by the red dashed line corresponds to results computed from the DNS database used to displace the synthetic particles.

investigate these trends we have considered a p.d.f. of velocity components from the PIV simulations $(\widetilde{U}$ and $\widetilde{V}$ ) and the DNS velocity fields $\left(\widetilde{U}_{D N S}\right.$ and $\left.\widetilde{V}_{D N S}\right)$ used to displace the synthetic particles. Results are plotted in figure 3, where encouraging agreement is observed between the SE- and DE-PIV simulations ( $\boldsymbol{\nabla}$, $\square$ and $\Delta$ symbols) and reference results from the DNS velocity field (red dashed line). Consequently, good agreement is also observed for the flow statistics of each velocity component to statistics computed directly from the DNS database. For example, the difference in the mean flow and turbulence intensities are within $0.1 \%$ and $0.5 \%$ respectively for both velocity components across all simulation cases.

\section{Experimental work}

\subsection{Description of experiments}

Based on our findings from the synthetic PIV simulations, a set of experiments are conducted in order to capture single-exposed (SE-PIV) and double-exposed (DE-PIV) images from specialist-PIV and consumer cameras, respectively. The experiments are performed in the High Reynolds Number Boundary Layer Wind Tunnel (HRNBLWT) at the University of Melbourne. The tunnel consists of a large development length of approximately $27 \mathrm{~m}$, offering the capability of achieving high $R e_{\tau}$ at relatively low free stream velocities. This provides a uniquely thick smooth-wall boundary layer at $R e_{\tau}$ of up to 25,000 , resulting in a larger measurable viscous length scale (and less acute spatial resolution issues).

Unlike most PIV experimental campaigns in the HRNBLWT (see works by [de Silva et al., 2014, Squire et al., 2014]), which are configured to achieve high $R e_{\tau}$ planar PIV databases, the present experiments are tailored to obtain snapshots of the large-scale motions in a turbulent boundary layer with high fidelity. Hence, the present experiments are conducted near the upstream end of the test section $(x \approx 5 \mathrm{~m})$, where the boundary layer thickness is small (a thickness of $\delta \approx 90 \mathrm{~mm}$ - still large relative to many boundary layer facilities, but small compared to what is achieved further downstream in the HRNBLWT). This enables us to capture well resolved instantaneous snapshots at moderate Reynolds number flows with a streamwise extent of $\sim 10 \delta$. We note the boundary layer thickness described in the preceding discussion, $\delta$, corresponds to the wall distance where the mean streamwise velocity is $99 \%$ of the freestream velocity.

Two sets of experiments are utilised in this work, namely, a SE-PIV experiment using an array of specialist PIV cameras, and a DE-PIV experiment using an array of consumer DSLR cameras. The SEPIV measurements consist of stitched images from eight 11 megapixel PCO4000 PIV cameras (see figure 4a), which provides velocity fields on a large streamwise/spanwise - hereafter $x y$ - plane. The solid red lines in figure 4(a) show the combined field of view, which spans $1.3 \mathrm{~m} \times 0.25 \mathrm{~m}$. The measurements are conducted at two freestream velocities $(10$ and $20 \mathrm{~m} / \mathrm{s})$, with corresponding Reynolds numbers ranges across the extent of the FOV of $R e_{\tau} \approx 2400-2900$ and $4800-5400$, respectively. We note the experiments are configured to capture $x y$ planes in the wake region of the flow $(z / \delta \approx 0.8)$ to facilitate the study of 
(a)
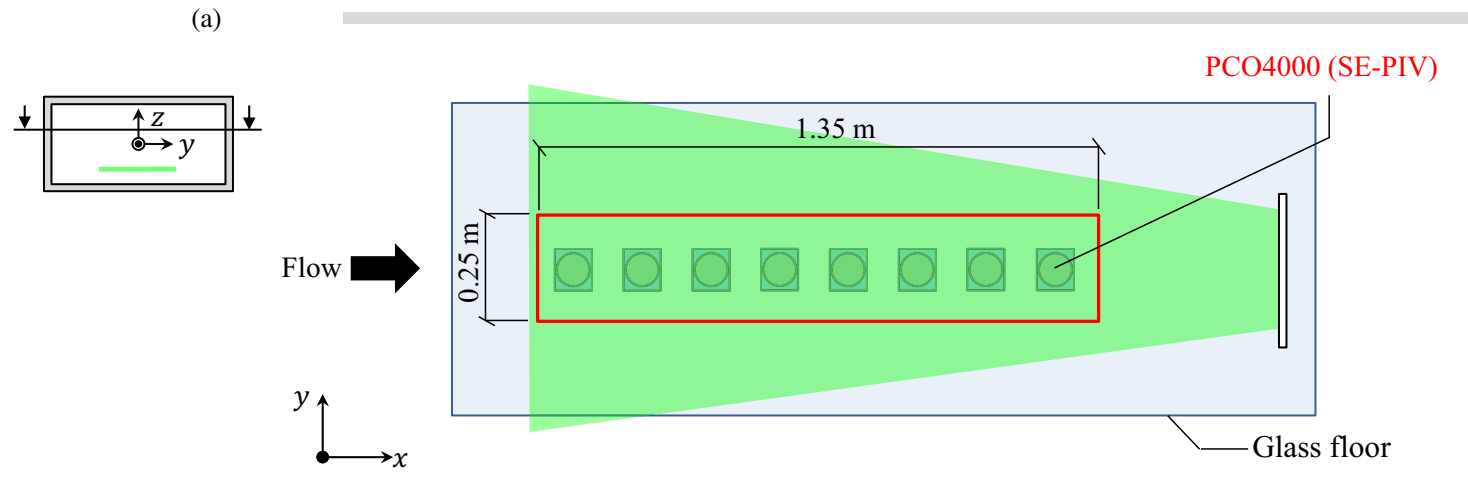

(b)

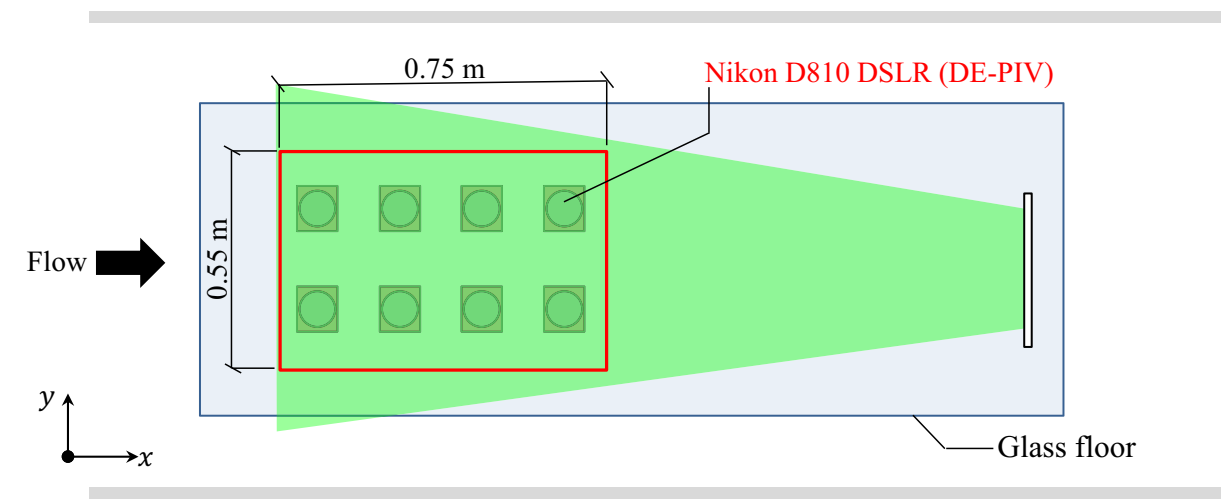

Fig. 4 Experimental setup used to conduct large field of view planar PIV experiments in the HRNBLWT over a streamwise/spanwise (xy) plane. The top (a) and bottom (b) panes show the configuration used for the SE-PIV and DE-PIV experiments respectively. The red solid line corresponds to the total field of view captured by the imaging system.

large-scale motions in the outer region of a turbulent boundary layer (results of this investigation are to be detailed in future works). A summary of the experimental and flow parameters are given in tables 2 and 3, respectively. Further details on the SE-PIV measurements can also be found in de Silva et al. [2015]. It should be noted that for consistency, $\delta^{+}\left(\sim R e_{\tau}\right)$ and $U_{\tau}$ are computed by applying the composite velocity profile of Chauhan et al. [2009] for all datasets. Additionally, as there is a slow growth in $\delta$ across the streamwise extent of the FOV, the laser sheet is tilted at a shallow angle to maintain a constant $z / \delta$ ratio across the entire FOV. Further, to maintain a constant light-sheet thickness along the full FOV a long focal length lens is combined with large distances between the light sheet optics [Reuther et al., 2015].

In order to facilitate direct comparisons, the DE-PIV experiments are configured to capture the same $x y$ plane examined by the SE-PIV experiments, and at equivalent Reynolds numbers. The experiments employ eight 36 megapixel Nikon D810 DSLR cameras (see figure 4b), which provide a combined spatial resolution of $\approx 0.3$ gigapixels. Additionally, to capitalise on the higher pixel density available, a larger FOV is captured, while still maintaining comparable spatial resolution (interrogation window size in viscous units).

Seeding for each experiment is injected into the wind tunnel in between the blower fan and the facility's flow conditioning section, which is then circulated throughout the whole laboratory to obtain a homogeneous seeding density across the test section. The illumination for the SE-PIV experiments was provided by a Spectra-Physics PIV-400 Nd:YAG laser, while the DE-PIV measurements used an Innolas Spitlight Compact $400 \mathrm{Nd:YAG} \mathrm{system.} \mathrm{In} \mathrm{both} \mathrm{measurement} \mathrm{configurations,} \mathrm{it} \mathrm{was} \mathrm{necessary} \mathrm{to} \mathrm{project} \mathrm{the} \mathrm{laser}$ sheet upstream through the working section to ensure adequate illumination across the large streamwise and spanwise extent of the FOV $(\approx 1 \mathrm{~m})$. Further details on the illumination configuration can be found in de Silva et al. [2015]. 


\begin{tabular}{|c|c|c|}
\hline & SE-PIV & DE-PIV \\
\hline $\begin{array}{l}\text { Flow medium } \\
\text { Seeding } \\
\text { Particle size } \\
\text { Acquisition frequency } \\
\text { Laser sheet thickness }\end{array}$ & \multicolumn{2}{|c|}{$\begin{array}{c}\text { Air } \\
\text { Polyamide particles } \\
\approx 1-2 \mu \mathrm{m} \\
1 \mathrm{~Hz} \\
\approx 1 \mathrm{~mm}\end{array}$} \\
\hline $\begin{array}{l}\text { Number of images } \\
\text { Total sensor resolution } \\
\text { Image pixel size } \\
\text { Field of view }(x \times y)\end{array}$ & $\begin{array}{l}1000 \\
\approx 90 \times 10^{6} \text { pixels } \\
\approx 65 \mu \mathrm{m} / \text { pixel } \\
\approx 1.35 \mathrm{~m} \times 0.25 \mathrm{~m}\end{array}$ & $\begin{array}{l}800 \\
\approx 290 \times 10^{6} \text { pixels } \\
\approx 35 \mu \mathrm{m} / \text { pixel } \\
\approx 0.75 \mathrm{~m} \times 0.55 \mathrm{~m}\end{array}$ \\
\hline
\end{tabular}

Table 2 Summary of parameters for the PIV experiments

\begin{tabular}{ccccccc}
\hline Symbol & Processing & \multirow{2}{*}{$U_{\infty}(\mathrm{m} / \mathrm{s})$} & $R e_{\tau}$ & $v / U_{\tau}(\mu \mathrm{m})$ & \multicolumn{2}{c}{$\Delta x \times \Delta y$} \\
& & & & & pixels & wall units \\
\hline $\boldsymbol{\Delta}$ & SE-PIV & \multirow{2}{*}{10} & \multirow{2}{*}{$2400-2900$} & \multirow{2}{*}{42} & $32 \times 32$ & $50 \times 50$ \\
& DE-PIV & & & & $64 \times 64$ & $50 \times 50$ \\
$\bullet$ & SE-PIV & \multirow{2}{*}{20} & \multirow{2}{*}{$4800-5400$} & 22 & $32 \times 32$ & $95 \times 95$ \\
& DE-PIV & & & & $64 \times 64$ & $95 \times 95$ \\
\hline
\end{tabular}

Table 3 Flow and processing parameters for the PIV experiments. All measurements are conducted at a wall-normal height of $z / \delta \approx$ 0.8. The friction velocity $U_{\tau}$ is estimated using the composite velocity profile of Chauhan et al. [2009].

\subsection{Image pre-processing and triggering of DSLR cameras for PIV}

The colour cameras deployed in the DE-PIV experiments contain a Bayer colour filter, and as a consequence, only $50 \%$ of the sensor's pixels are sensitive to the $532 \mathrm{~nm}$ wavelength emitted from the laser. When processing digital colour images, a demosaicing algorithm is routinely used to interpolate colour channel information across every pixel and utilise the full resolution of the sensor. Under the $532 \mathrm{~nm}$ green laser light of our PIV experiments, the intensities of pixels sensitive to red and blue light are enhanced during the demosaicing process. We observed best results using the AMAZE demosaicing algorithm [Martinec and Lee, 2010], which contained no significant artefacts in the PIV images. However, good performance of the algorithm requires average particle image sizes to be at least a few pixels in diameter.

External multi-camera synchronisation and triggering are not typically standard features of the consumer cameras used in the present DE-PIV experiments, and this subject therefore warrants some discussion. In the present case, due to the modest acquisition rate $(1 \mathrm{~Hz})$, each camera is equipped with a wireless remote shutter adapter (SMDV RFN-4s). These adapters on each camera are then simultaneously triggered via a single wireless transmitter, which is interfaced (via some relatively simple custom electronics) to a standard delay generator box to synchronise the cameras with the pulsed laser operation. The aforementioned trigger mechanism, coupled with a long exposure time ( 0.5 seconds) and high-speed CompactFlash card for storage, enabled reliable continuous capture of double exposed images at $1 \mathrm{~Hz}$. We note that, although not pursued in the present work, higher capture rates are also possible.

\subsection{Calibration and PIV evaluation}

Because multiple cameras are used in the present experiments to construct a large FOV, a calibration procedure is essential to stitch the velocity fields from each camera together and also to account for image distortions. In the present experiments, we employ a large calibration target that spans the entire extent of the FOV, which has been proven to work well for similar large-FOV experiments in the same facility (see de Silva et al. [2012b, 2014]). The target enables us to apply a pixel-to-real space conversion by mapping the pixel positions from each camera to real space coordinates within the wind tunnel. Additionally, we are able to precisely locate each camera's FOV relative to one another, which is necessary when stitching the individual vector fields from each camera together.

Similar to the simulation work presented in section 2, the experimental PIV images are processed using an in-house PIV package developed at the University of Melbourne [de Silva et al., 2014, Squire et al., 

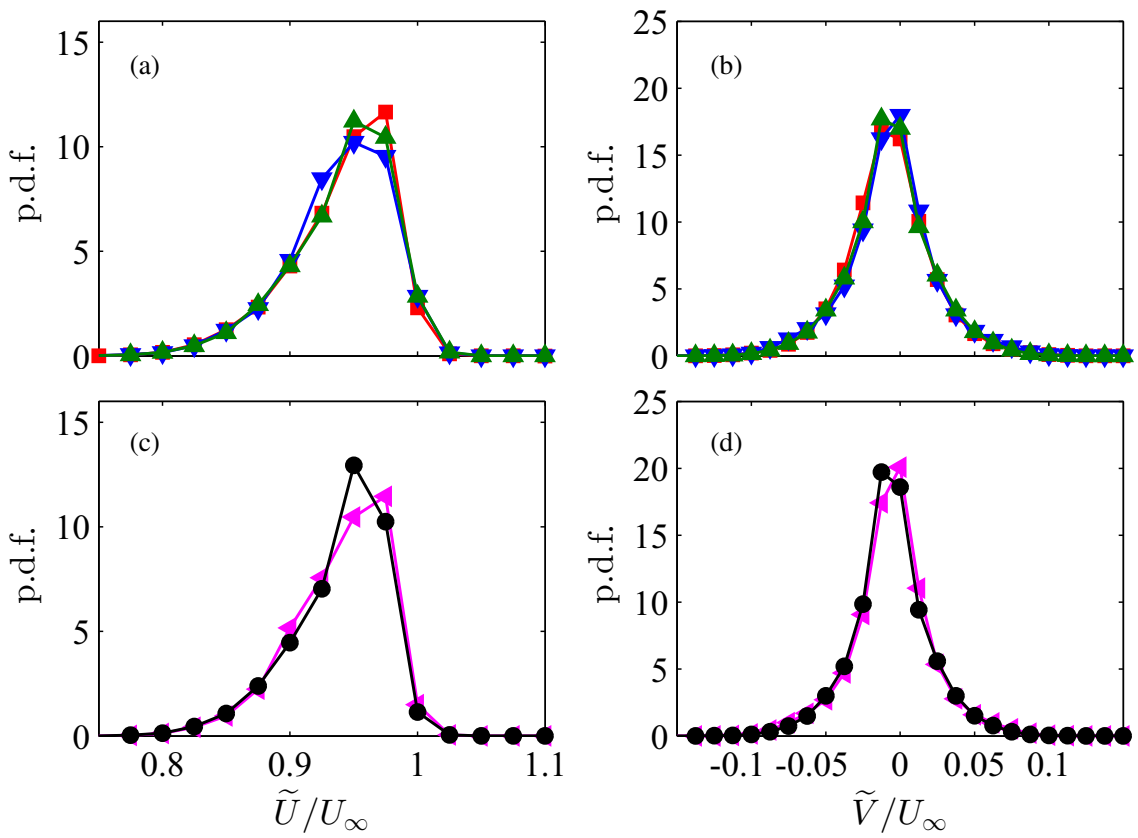

Fig. 5 P.d.f. of the (a and c) streamwise $(\widetilde{U})$ and (b and d) spanwise $(\widetilde{V})$ velocity components from the experimental databases at $z / \delta \approx 0.8$. The top pane (a-b) corresponds to results at $R e_{\tau} \approx 2500$ and the bottom pane (c-d) corresponds to results at $R e_{\tau} \approx 5000$. The $\boldsymbol{\nabla}, \triangleleft$ symbols correspond to SE-PIV, and $\boldsymbol{\Lambda}$, symbols correspond to DE-PIV experiments. (a-b) symbols correspond to results computed from a DNS database at matched $R e$ and wall-normal location.

2014]. Further, the velocity vectors are evaluated using the same cross-correlation algorithm, with multi-grid [Willert, 1997] and window deformation [Scarano, 2001]. The databases are processed with 50\% overlap and the final interrogation window sizes for each dataset are detailed in table 3 . It should be noted that we utilise a low magnification level, therefore the particle image sizes are typically $\sim 2$ pixels (in particular for the SE-PIV databases). This size is less than that typically recommended for PIV experiments [Raffel et al., 2017], and therefore can lead to some degree of pixel-locking in the velocity fields (a bias error towards integer values) [Westerweel, 1997]. To minimise this shortcoming, a Gaussian kernel filter is applied to the raw images [Adrian and Westerweel, 2011]. Additionally, the window deformation technique used during vector evaluation is also known to further reduce the influence of pixel-locking [Scarano, 2001].

\subsection{Experimental validation}

In order to assess the quality of the DE-PIV measurements captured from the DSLR camera array, flow statistics are compared to prior well-resolved SE-PIV measurements, and to a DNS database in a turbulent boundary layer at matched $R e_{\tau}$. Accordingly, figure 5(a) presents a p.d.f. of the streamwise $(\widetilde{U})$ and spanwise $(\widetilde{V})$ velocity components from the large FOV SE-PIV ( $\nabla$ symbols) and DE-PIV ( $\boldsymbol{\Delta}$ symbols) measurements. Results are presented at $R e_{\tau} \approx 2500$, at a fixed wall-normal location of $z / \delta \approx 0.8$. Further, statistics computed from a DNS database at matched $R e_{\tau}$ are also included ( $\square$ symbols). To facilitate a direct comparison, results from the DNS database are presented at a wall-normal location where the ratio $\bar{U} / U_{\infty}$ is equivalent to those from the experiments. Therefore, the results from the DNS databases can be used to benchmark the quality of the experiments, isolating any experimental uncertainties which are not present in DNS. The p.d.f.'s of $\widetilde{U}$ and $\widetilde{V}$ plotted in 5(a) shows good agreement between the experiments and the reference DNS database. More specifically, the difference in the mean flow and turbulence intensities between the experiments (SE- and DE-PIV) and DNS results for both velocity components are within $0.2 \%$ and 2\%, respectively. Therefore, similar to our findings from the PIV simulations in section 2 (see figure 3 ) the experiments also reveal that velocity fields with comparable accuracy (within the uncertainty of the measurement) can be obtained from a cost-effective, high-resolution consumer camera array operated to perform DE-PIV. 
(a)
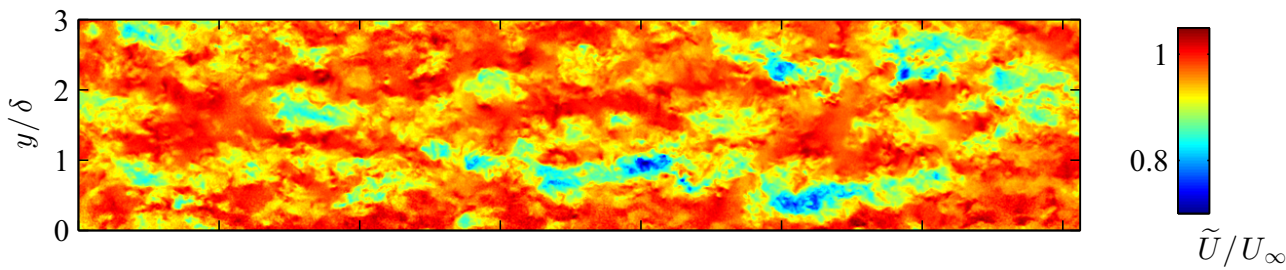

(b)

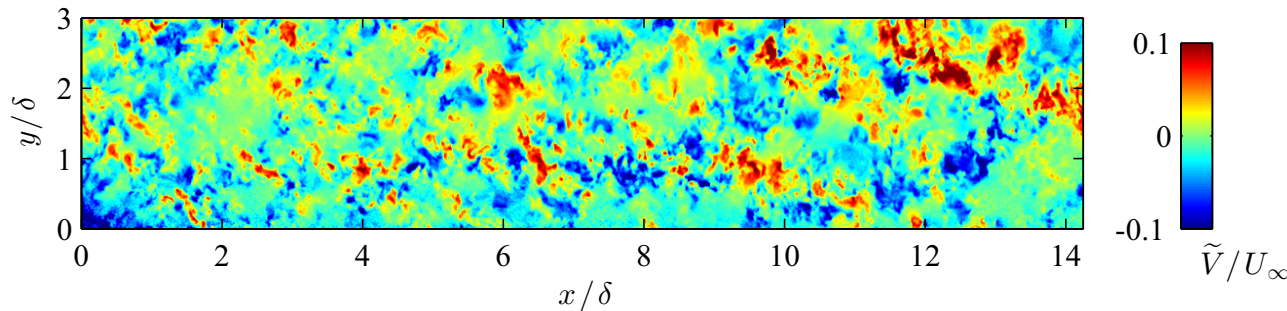

(c)
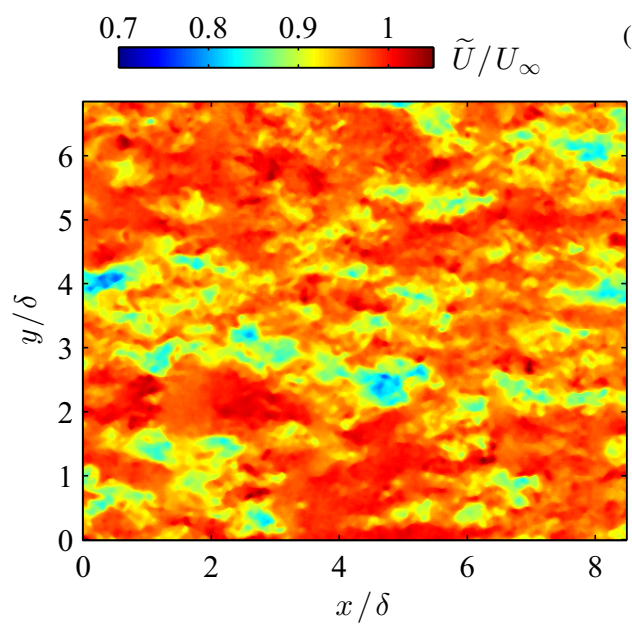

(d)
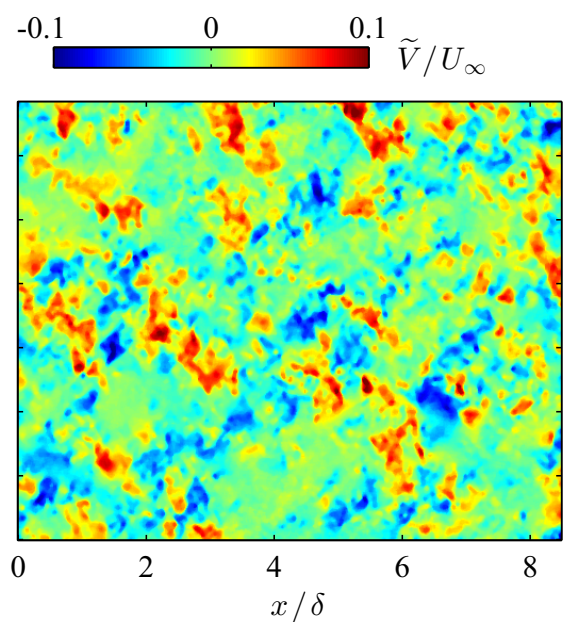

Fig. 6 Instantaneous velocity fields from the PIV datasets from the (a-b) SE-PIV and (c-d) DE-PIV experiments. Results are shown at $R e_{\tau} \approx 2500$ on a $x y$ plane located in the wake region of the flow $(z / \delta \approx 0.8)$. Colour contours represent the instantaneous velocity components captured for each configuration, where (a,c) shows streamwise velocity, and (b,d) shows spanwise velocity. Spatial extents are normalised by $\delta$, computed at the middle of each field of view.

Figures 5(c-d) present the same flow statistics as in 5(a-b) at $R e_{\tau} \approx 5000\left(U_{\infty} \approx 20 \mathrm{~m} / \mathrm{s}\right)$, measured at a wall-normal location of $z / \delta \approx 0.8$. Due to the lack of DNS databases at higher $R e_{\tau}$, comparisons are only shown between the SE- and DE-PIV measurements. The results show good agreement between the SE- and DE-PIV databases to those obtained from the databases at lower $R e_{\tau}$. It is important to note that both the $R e_{\tau} \approx 2500$ and $R e_{\tau} \approx 5000$ datasets are processed using the same interrogation window size in pixels. Therefore we expect larger spatial attenuation for flow statistics at $U_{\infty} \approx 20 \mathrm{~m} / \mathrm{s}$ compared to results presented at $U_{\infty} \approx 10 \mathrm{~m} / \mathrm{s}$.

\subsection{Instantaneous velocity fields and spectra}

The large spatial extent of the current measurements enables the visualisation of large coherent motions known to exist in boundary layers at moderate/high Reynolds numbers. To illustrate the value of this flow visualisation, sample instantaneous velocity fields are presented in figure 6, where (a-b) and (c-d) show $x y$ planar velocity fields captured using SE- and DE-PIV, respectively. Colour contours correspond to the streamwise and spanwise velocity components. Visual inspection of these velocity fields reveals spatial structures over a wide range of length scales, emphasising the importance of large-FOV PIV measurements with good spatial resolution. For example, the velocity fields show several characteristic features of the wake region in boundary layer flows. These include patches of turbulent bulges (see figure 6a,c), which have 


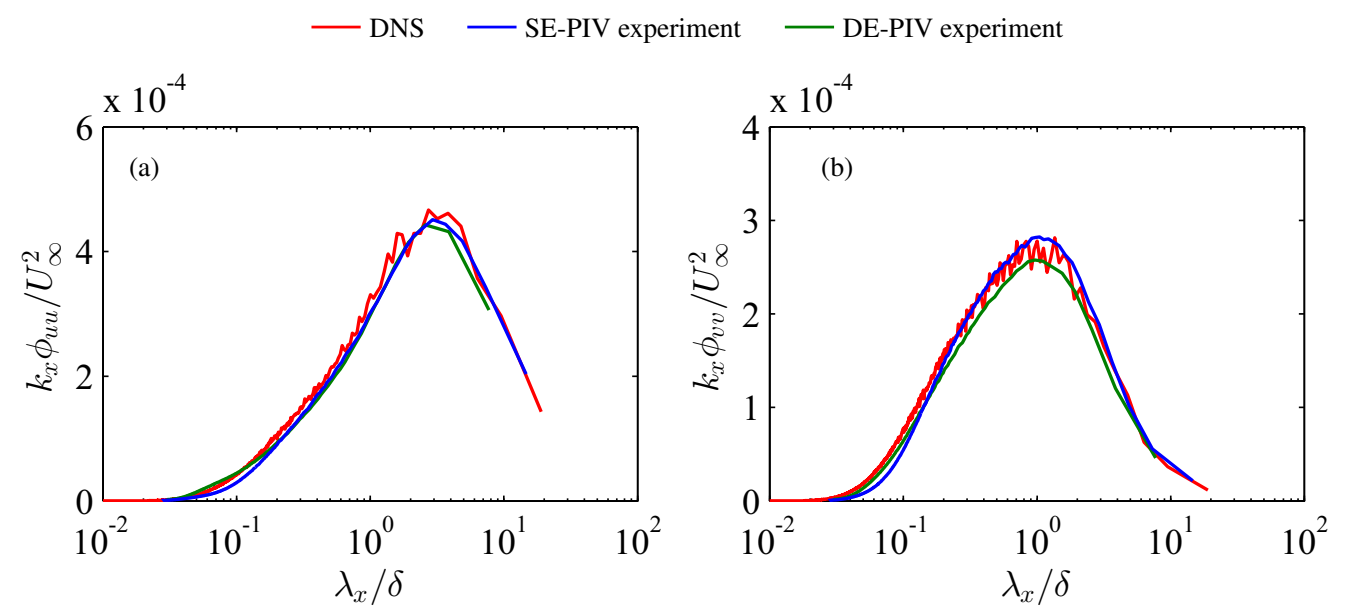

Fig. 7 Comparison of the pre-multiplied energy spectra between the PIV experiments at $R e_{\tau} \approx 2500$ and DNS databases. Results are shown as a function of streamwise wavelength $\lambda_{x}$ for the (a) streamwise and (b) spanwise velocity fluctuations. The solid blue and green lines correspond to results from the SE-PIV and DE-PIV experimental databases, respectively. The solid red line shows results computed from a DNS database at matched $R e$ and wall-normal location.

lengths on the order of the boundary layer thickness. The wider velocity fields (larger spanwise domain) captured using the DE-PIV (see figure 6d) also clearly show oblique patterns in the spanwise coherence that are oriented at approximately $\pm 45^{\circ}$, dependent on the sign of $\widetilde{V}$ [Sillero et al., 2014].

Another notable advantage of high-resolution PIV databases obtained using a multi-camera array is the large dynamic spatial range that can be captured [Adrian, 1997, Westerweel et al., 2013], which is critical to the measurement of high Reynolds number flows. Consequently, these databases can be used to directly compute the energy spectra of the turbulent fluctuations in the boundary layer. Results from the experimental databases at $R e_{\tau} \approx 2500$ are shown in figure 7, where (a) and (b) correspond to the pre-multiplied energy spectra of the streamwise and spanwise velocity fluctuations, respectively. No discernible differences are observed between the SE-, DE-PIV, and the reference DNS databases, indicating that both experiments have accurately captured the turbulent scales that coexist in the flow. Moreover, no significant increase in energy at the smaller wavelengths is observed (even from the DE-PIV measurements). Therefore, the additional measurement noise present by employing double-exposed images does not appear to significantly contaminate the turbulent scales present in the flow, and is likely to only manifest at smaller wavelengths (see Atkinson et al. [2014]).

\section{Conclusions}

In this study, we describe large-scale multi-camera planar PIV measurements, tailored to capture the wide range of scales prevalent in high Reynolds number $(R e)$ turbulent boundary layers. To this end, specialist PIV cameras are substituted with modern consumer full-frame DSLR cameras, which are typically available at a fraction of the cost $(\approx 1 / 10$ th the cost per megapixel). These cameras are configured to capture double-exposed PIV images (DE-PIV), which are shown to be well-suited to perform high-resolution PIV measurements in turbulent boundary layers, where directional ambiguity is negligible.

Through a simulation study of PIV, it is shown that despite a loss-of-accuracy from DE-PIV when compared to SE-PIV (see also Keane and Adrian [1992]) at matched conditions, the greater resolution available by using consumer-cameras provides more comparable accuracy to those obtained from limited-resolution SE-PIV. Based on these findings, a set of DE-PIV experiments are conducted with an effective total sensor resolution of $\sim 0.3$ gigapixels. Comparison of flow statistics with large FOV SE-PIV measurements using specialist PIV cameras, and to DNS databases at matched $R e_{\tau}$, revealed that velocity fields with comparable accuracy can be captured using a cost-effective high-resolution DSLR camera array with DE-PIV. Moreover, comparing the pre-multiplied energy spectra of the streamwise and spanwise velocity fluctuations from the experiments with the reference numerical database also showed good agreement. These observa- 
tions confirm that a significant proportion of the spatial scales that coexist in the studied boundary layer have been accurately captured.

Ultimately, the present work provides a foundation for utilising DE-PIV in large-scale PIV measurements of high Reynolds number boundary layers, where well-resolved PIV measurements would otherwise be considered economically infeasible. Such experiments will be able to capture direct spatial information of the largest coherent motions prevalent in boundary layers and to unravel their dynamics and associated Reynolds number trends. The enhanced cost-effectiveness of the use of a consumer DSLR camera array, with further refinement, also has far-reaching potential for industrial applications. In using these techniques, spatial resolution may not need to be sacrificed in order to balance economic feasibility and quantitative results.

Acknowledgements The authors wish to gratefully thank the financial support of the Australian Research Council.

\section{References}

R. J. Adrian. Image shifting technique to resolve directional ambiguity in double-pulsed velocimetry. App. Optics, 25(21):3855-3858, 1986.

R. J. Adrian. Dynamic ranges of velocity and spatial resolution of particle image velocimetry. Meas. Sci. Tech., 8(12):1393, 1997.

R. J. Adrian, C. D. Meinhart, and C. D. Tomkins. Vortex organization in the outer region of the turbulent boundary layer. J. Fluid Mech., 422:1-54, 2000.

R. J. Adrian and J. Westerweel. Particle Image Velcimetry. Cambridge University Press, 2011.

C. Atkinson, N. A. Buchmann, and J. Soria. An experimental investigation of turbulent convection velocities in a turbulent boundary layer. Flow, Turb. Comb., pages 1-17, 2014.

H. H. Bruun. Hot-Wire Anemometry: Principles and Signal Analysis. Oxford University Press, 1995.

N. A. Buchmann, Y. C. Kücükosman, K. Ehrenfried, and C. J. Kähler. Wall pressure signature in compressible turbulent boundary layers. In Prog. Wall Turb. 2, pages 93-102. Springer, 2016.

K. A. Chauhan, P. A. Monkewitz, and H. M. Nagib. Criteria for assessing experiments in zero pressure gradient boundary layers. Fluid. Dyn. Res., 41(2):021404, 2009.

C. M. de Silva, R. Baidya, M. Khashehchi, and I. Marusic. Assessment of tomographic PIV in wall-bounded turbulence using direct numerical simulation data. Exp. Fluids, 52(2):425-440, 2012 a.

C. M. de Silva, K. A. Chauhan, C. H. Atkinson, N. A. Buchmann, N. Hutchins, J. Soria, and I. Marusic. Implementation of large scale PIV measurements for wall bounded turbulence at high Reynolds numbers. In $18^{\text {th }}$ Aust. Fluid Mech. Conf., volume 18, pages 308-311, Launcheston, Australia, 2012b.

C. M. de Silva, E.P. Gnanamanickam, C. Atkinson, N. A. Buchmann, N. Hutchins, J. Soria, and I. Marusic. High spatial range velocity measurements in a high Reynolds number turbulent boundary layer. Phys. Fluids, 26(2):025117, 2014.

C. M. de Silva, D. T. Squire, N. Hutchins, and I. Marusic. Towards capturing large scale coherent structures in boundary layers using particle image velocimetry. In Proc. 6th Aust. Conf. Laser Diag. Fluid Mech. Comb., pages 1-4. University of Melbourne, 2015.

S. Funatani, T. Takeda, and K. Toriyama. High-resolution three-color PIV technique using a digital SLR camera. J. Flow Vis. Im. Proc., 20(1-2), 2013.

K. Grayson, C. M. de Silva, N. Hutchins, and I. Marusic. Impact of mismatched and misaligned laser light sheet profiles on piv performance. Exp. Fluids, 59(1):2, 2018.

C. J. Kähler, T. Astarita, P. P. Vlachos, J. Sakakibara, R. Hain, S. Discetti, R. La Foy, and C. Cierpka. Main results of the 4th international PIV challenge. Exp. Fluids, 57(6):97, 2016.

C. J. Kähler, S. Scharnowski, and C. Cierpka. High resolution velocity profile measurements in turbulent boundary layers. In $16^{\text {th }}$ Int. Symp. App. Laser Tech. Fluid Mech., Lisbon, Portugal, 09-12 July, 2012a.

C. J. Kähler, S. Scharnowski, and C. Cierpka. On the resolution limit of digital particle image velocimetry. Exp. Fluids, 52(6):1629-1639, $2012 \mathrm{~b}$.

R. D. Keane and R. J. Adrian. Optimization of particle image velocimeters. part: I. double pulsed systems. Meas. Sci. Technol., 1(11):1202, 1990.

R. D. Keane and R. J. Adrian. Theory of cross-correlation of PIV images. Appl Sci Res, 49:191-215, 1992. 
T. Knopp, D. Schanz, A. Schröder, M. Dumitra, C. Cierpka, R. Hain, and C. J. Kähler. Experimental investigation of the log-law for an adverse pressure gradient turbulent boundary layer flow at $\operatorname{Re}_{\theta}=10000$. Flow, Turb. Comb., 92(1-2):451-471, 2014.

J. H. Lee, Kevin, J. P. Monty, and N. Hutchins. Validating under-resolved turbulence intensities for PIV experiments in canonical wall-bounded turbulence. Exp. Fluids, 57(8):129, 2016.

E. Martinec and P. Lee. Amaze demosaicing algorithm, 2010.

M. Raffel and J. Kompenhans. Theoretical and experimental aspects of image-shifting by means of a rotating mirror system for particle image velocimetry. Meas. Sci. Tech., 6(6):795, 1995.

M. Raffel, C. E. Willert, F. Scarano, C. J. Kähler, S. Wereley, and J. Kompenhans. Particle Image Velocimetry - A Practical Guide. Springer Berlin Heidelberg New York, 2017.

N. J. Reuther, D. Schanz, S. Scharnowski, R. Hain, A. Schröder, and C. Kähler. Experimental investigation of adverse pressure gradient turbulent boundary layers by means of large-scale PIV. In Proc. $11^{\text {th }}$ Int. Symp. Part. Image Vel., volume 10, Santa Barbara, USA, 2015.

F. Scarano. Iterative image deformation methods in PIV. Meas Sci Tech., 13:R1-19, 2001.

J. A. Sillero, J. Jiménez, and R. D. Moser. One-point statistics for turbulent wall-bounded flows at Reynolds numbers up to $\delta^{+}=2000$. Phys. Fluids, 25(10):105102, 2013.

J. A. Sillero, J. Jiménez, and R. D. Moser. Two-point statistics for turbulent boundary layers and channels at Reynolds numbers up to $\delta^{+}=2000$. Phys. Fluids, 26(10):105109, 2014.

D. T. Squire, C. M. de Silva, N. Hutchins, and I. Marusic. High fidelity spatial measurements at high Reynolds numbers in turbulent boudary layers. In $17^{\text {th }}$ Int. Symp. App. Laser Tech. Fluid Mech., Lisbon, Portugal, 2014.

Z. Wang, Q. Gao, and J. Wang. A triple-exposure color PIV technique for pressure reconstruction. Sci.China Technol. Sci., 60(1):1-15, 2017.

J. Westerweel. Fundamentals of digital particle image velocimetry. Meas Sci Tech., 8:1379-1392, 1997.

J. Westerweel, G. E. Elsinga, and R. J. Adrian. Particle image velocimetry for complex and turbulent flows. Ann. Rev. Fluid Mech., 45:409-436, 2013.

C. Willert. Stereoscopic digital particle image velocimetry for application in wind tunnel flows. Meas. Sci. Tech., 8(12):1465, 1997.

N. A. Worth, T. B. Nickels, and N. Swaminathan. A tomographic PIV resolution study based on homogeneous isotropic turbulence DNS data. Exp. Fluids, 49:637-656, 2010. 


\section{University Library}

\section{- M M I N E R VA \\ A gateway to Melbourne's research publications}

Minerva Access is the Institutional Repository of The University of Melbourne

Author/s:

de Silva, CM;Grayson, K;Scharnowski, S;Kaehler, CJ;Hutchins, N;Marusic, I

Title:

Towards fully-resolved PIV measurements in high Reynolds number turbulent boundary layers with DSLR cameras

Date:

2018-06-01

Citation:

de Silva, C. M., Grayson, K., Scharnowski, S., Kaehler, C. J., Hutchins, N. \& Marusic, I. (2018). Towards fully-resolved PIV measurements in high Reynolds number turbulent boundary layers with DSLR cameras. JOURNAL OF VISUALIZATION, 21 (3), pp.369-379. https://doi.org/10.1007/s12650-017-0465-z.

Persistent Link:

http://hdl.handle.net/11343/282580 